\title{
Efecto del incumplimiento de la hipótesis de normalidad en los gráficos de control de la media
}

\author{
MOYA FERNÁNDEZ, PABLO \\ Universidad de Granada \\ Correo electrónico: pjmoyafernandez@ugr.es \\ ÁlVAREZ-VERDEJO, ENCARNACIÓN \\ Universidad de Granada \\ Correo electrónico: encarniav@ugr.es \\ BLANCO-ENCOMIENDA, FRANCISCO JAVIER \\ Universidad de Granada \\ Correo electrónico: jble@ugr.es
}

\begin{abstract}
RESUMEN
Los gráficos de control son ampliamente usados para monitorizar la calidad de procesos industriales. Tradicionalmente se asume que la variable aleatoria que representa la característica de calidad se distribuye de forma normal y los límites de control se definen de forma que la probabilidad de obtener una falsa alarma es 0.0027. Sin embargo, en la práctica la característica de calidad podría seguir otra distribución y este hecho podría afectar a la eficiencia del gráfico de control.

En el presente trabajo se realiza un estudio de simulación Monte Carlo con el objetivo de evaluar empíricamente el impacto del incumplimiento del supuesto de normalidad en el gráfico de control para la media. Se consideran distintas distribuciones probabilísticas para analizar diferentes grados de incumplimiento. Adicionalmente, se han considerado situaciones en los que el proceso está bajo control y fuera de control.

Los resultados sugieren que los gráficos de control son una herramienta efectiva cuando la distribución de la característica de calidad tiene una leve asimetría. Sin embargo, para obtener una efectividad similar a la obtenida bajo normalidad es necesario aumentar levemente el número de muestras o el tamaño de las mismas. En el caso de que la característica de calidad siga una distribución con un grado de asimetría mayor es necesario aumentar los tamaños muestrales para obtener resultados aceptables. Por último, no es recomendable utilizar los gráficos de control en situaciones extremas de falta de normalidad.

Palabras clave: control estadístico de procesos, simulación Monte Carlo, longitud media de las rachas, error tipo I, monitorizar.
\end{abstract}

Clasificación JEL: M11.

MSC2010: 62P30.

Artículo recibido el 9 de septiembre de 2019 y aceptado el 9 de diciembre de 2019. 


\title{
Effect of non-compliance with the normality hypothesis on the mean control charts
}

\begin{abstract}
Control charts are widely used to monitor the quality of industrial processes. It is quite common to assume that the random variable associated to the quality characteristic has a Normal distribution, and the control limits are defined so that the probability of obtaining a false alarm is 0.0027 . However, the quality characteristic could follow a different distribution in practice, and this fact could have an impact on the efficiency of the control chart.

In this paper, a Monte Carlo simulation study is carried out to evaluate empirically the impact of the lack of the normality assumption on the control chart for the mean. Different probabilistic distributions are considered. In addition, under control and out of control processes are considered.

The results derived from the simulation study suggest that control charts are an effective tool when the distribution of the quality characteristic is slightly asymmetric. However, a large number of samples or larger sample sizes are required to obtain similar results to the case of symmetric distributions. In the case of asymmetric distributions, it is necessary to increase the sample sizes to obtain acceptable results. Finally, control charts are not recommended under evident cases of non-normality.
\end{abstract}

Keywords: statistical process control, Monte Carlo simulation, average run length, type I error, monitor.

JEL classification: M11.

MSC2010: 62P30.

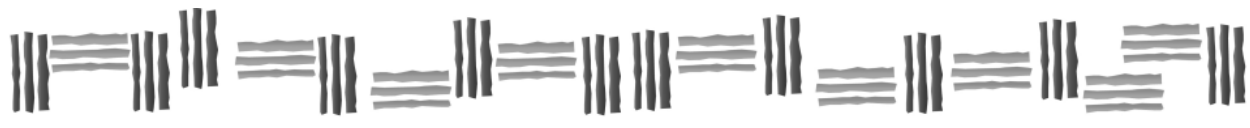




\section{Introducción.}

En la fase de producción o fabricación de un producto pueden surgir distintas fuentes de variabilidad que pueden alterar la calidad del producto. Esta variabilidad se puede clasificar en: (i) variabilidad debida a causas fortuitas y (ii) variabilidad debida a causas asignables. El objetivo principal del control estadístico de procesos es la reducción de la variabilidad debida a causas asignables. Puede considerarse que los gráficos de control, desarrollados por Shewhart (1931), son una de las técnicas cuantitativas más simples y efectivas que puede reducir al mínimo la variabilidad de un proceso.

Los gráficos de control se utilizan para detectar cambios en un proceso de fabricación, evaluar los parámetros del proceso, así como una aproximación de su capacidad o rendimiento para producir productos de acuerdo a las especificaciones establecidas.

Los gráficos de control de la media son los que controlan el valor medio del proceso, es decir, permiten determinar si la media de una determinada característica de calidad y en determinado artículo se encuentra o no dentro de los límites de control fijados de antemano. La metodología general para la obtención de un gráfico de control de la media se expone a continuación.

Supongamos que la característica de la calidad estudiada tiene distribución Normal, es decir, $x \rightarrow N(\mu, \sigma)$, donde $\mu$ es la media del proceso y $\sigma$ es la desviación típica del mismo. En líneas generales, los gráficos de control se obtienen seleccionando distintas muestras y representando en dicho gráfico el valor de un determinado estadístico. El estadístico que se representa depende del parámetro del proceso que se controla. Por ejemplo, la media del proceso se puede controlar mediante el gráfico de control de la media, en el que se representan las medias muestrales:

$$
\bar{x}_{i}=\frac{1}{n} \sum_{j=1}^{n} x_{i j}
$$

obtenidas a partir de muestras de tamaño constante $n$ y tomadas secuencialmente, donde $x_{i j}$ denota el valor de la característica de la calidad para el producto $j$-ésimo en la muestra $i$-ésima.

Los gráficos de control constan de una línea central y dos límites de control situados a $k$ desviaciones típicas de la línea central, que permiten determinar si el proceso se encuentra bajo control o fuera de control; así, el proceso estará bajo control si el valor del estadístico se encuentra entre los límites de control y se encontrará fuera de control en otro caso. Que el proceso se encuentre fuera de control significa que en dicho proceso existen causas asignables, tales como errores de los empleados presentes en la producción que impiden que los productos que se están produciendo cumplan con las especificaciones fijadas. En el lado opuesto, que el proceso esté bajo control indica que los productos, en su mayoría, sí cumplen con las especificaciones establecidas, aunque pueden existir otras causas inevitables, debidas al azar, que pueden provocar pequeñas variaciones en la calidad de los productos. A modo de ejemplo, en la Figura 1 se presenta un gráfico de control para la media. 


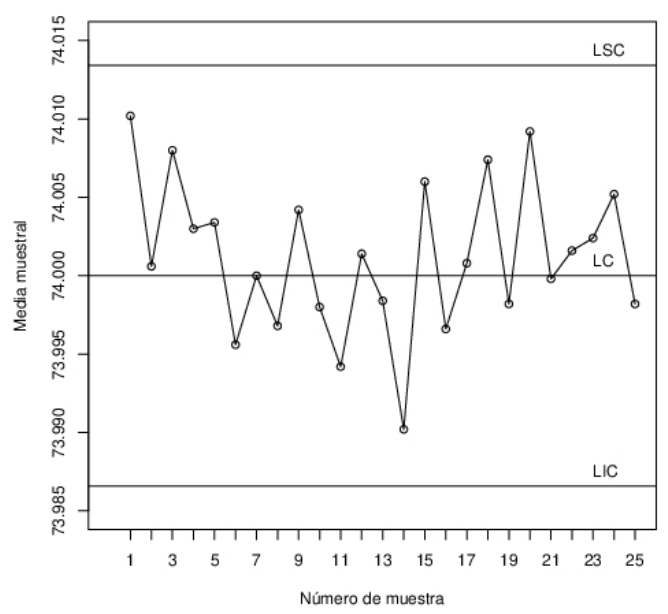

Fuente: Datos reales tomados de la base de datos "pistonrings" incluidos en la librería "qce" (Scrucca, 2004) del software estadístico R.

Cuando se conocen los parámetros $\mu$ (media real del proceso) y $\sigma$ (desviación típica real del proceso) los límites de control vienen dados por:

$$
L I C=\mu-k \frac{\sigma}{\sqrt{n}} ; L C=\mu ; L S C=\mu+k \frac{\sigma}{\sqrt{n}}
$$

donde $L I C$ es el denominado Límite Inferior de Control, $L C$ es la denominada Línea Central y $L S C$ es el Límite Superior de Control. Cabe decir que el criterio de $k=3$ es el que se considera habitualmente en la práctica (Abbas et al., 2011; Chen, 1997; Montgomery, 2009; Mitra, 2016). Se puede observar que los límites de control dependen de los parámetros $\mu$ y $\sigma$, que se han asumido conocidos. Sin embargo, los parámetros del modelo son generalmente desconocidos y en tal caso es necesario estimarlos utilizando la información muestral (Saleh et al., 2015). Los límites de control se estiman mediante el uso de $m$ muestras, con tamaño $n$, y tomadas cuando se cree que el proceso está bajo control. Las expresiones de los límites de control en el caso de parámetros desconocidos son:

$$
L \hat{I} C=\overline{\bar{x}}-k \frac{\hat{\sigma}}{\sqrt{n}} ; \hat{L} C=\overline{\bar{x}} ; L \hat{S} C=\overline{\bar{x}}+k \frac{\hat{\sigma}}{\sqrt{n}}
$$

donde $\overline{\bar{x}}$ es un estimador del parámetro $\mu$ definido como

$$
\overline{\bar{x}}=\frac{1}{m} \sum_{i=1}^{m} \bar{x}_{i}
$$

es decir, $\overline{\bar{x}}$ es la media aritmética de las $m$ medias muestrales. Por su parte, $\hat{\sigma}$ es un estimador de $\sigma$. En la práctica, los métodos más conocidos y utilizados para estimar el parámetro $\sigma$ son:

$$
\hat{\sigma}_{S}=\frac{\bar{S}}{c_{4}[n]} ; \quad \hat{\sigma}_{R}=\frac{\bar{R}}{d_{2}[n]} ; \hat{\sigma}_{p}=\frac{S_{p}}{c_{4}[1+m(n-1)]}
$$

donde $\bar{S}=\frac{1}{m} \sum_{i=1}^{m} S_{i}$ es la media de las desviaciones típicas de las muestras, que se definen como 


$$
\begin{gathered}
S_{i}=\left(\frac{1}{n-1} \sum_{j=1}^{n}\left(x_{i j}-\bar{x}_{i}\right)^{2}\right)^{1 / 2}, \\
\bar{R}=\frac{1}{m} \sum_{i=1}^{m} R_{i} \text { es el promedio de los rangos } R_{i}=\max \left(x_{i j}\right)-\min \left(x_{i j}\right) \mathrm{y} \\
S_{p}=\left(\frac{1}{m} \sum_{i=1}^{m} S_{i}^{2}\right)^{1 / 2} .
\end{gathered}
$$

$c_{4}[\cdot]$ y $d_{2}[\cdot]$ son constantes que dependen de los tamaños muestrales. En el anexo VI de Montgomery (2009) pueden consultarse tablas para estas constantes y para diversos valores de $n$. La elección del estimador a usar depende del tamaño de las sucesivas muestras que se toman para controlar la característica de calidad del proceso, es decir, $n$. Por ejemplo, Woodall y Montgomery (2000) desaconsejan el estimador $\hat{\sigma}_{R}$ para muestras con tamaños $n \geq 10$; $\sin$ embargo, este estimador tiene un buen comportamiento en muestras pequeñas $(n \leq 6)$.

Un supuesto básico de los gráficos de control es la hipótesis de normalidad en la variable que tiene un seguimiento de calidad. De hecho, los gráficos de control están basados en medias, por lo que para muestras elevadas se puede asumir normalidad teniendo en cuenta el Teorema Central del Límite. Sin embargo, en la práctica se consideran muestras pequeñas de tamaño en torno a 5 unidades. El objetivo principal de este trabajo es analizar el efecto de la falta o incumplimiento de la hipótesis de normalidad en el comportamiento del gráfico de control de la media. Estudios similares sobre la falta de normalidad han sido discutidos por varios autores (Chan et al., 1988; Schilling \& Nelson, 1976; Yourstone \& Zimmer, 1992), pero en ningún caso se ha llevado a cabo un análisis profundo de este problema mediante estudios de simulación Monte Carlo.

En este trabajo se asumen las distribuciones Normal, Gamma y Uniforme con el fin de comparar el comportamiento de los correspondientes gráficos de control de la media. La distribución Normal se considera puesto que es la hipótesis básica asumida en la mayoría de las aportaciones en los gráficos de control. La distribución Gamma es otra distribución continua muy conocida y utilizada que puede llegar a tener una importante asimetría hacia la derecha. Se consideran características de calidad con distintas varianzas con el objetivo de analizar distribuciones Gamma con distintos grados de asimetría. Por último, se considera la distribución Uniforme como un caso extremo ante la falta de normalidad con la pretensión de extraer conclusiones en cuanto al comportamiento de los gráficos de control de la media en esta situación extrema.

El resto del trabajo se estructura como sigue. En la Sección 2 se describe el estudio de simulación Monte Carlo llevado a cabo. En primer lugar, se definen las medidas empíricas que han sido utilizadas para analizar la eficiencia de los gráficos de control. A continuación, se describen de forma detallada los diferentes escenarios que se han considerado. Los resultados más relevantes derivados del estudio de simulación se muestran en la Sección 3. Por último, se exponen las principales conclusiones en la Sección 4.

\section{Estudio del efecto del incumplimiento de normalidad mediante simulación Monte Carlo.}

En líneas generales, el objetivo de un estudio de simulación Monte Carlo es evaluar el comportamiento de una determinada técnica de estimación o procedimiento estadístico mediante la valoración o comportamiento medio de dicho método cuando se replica o se pone a prueba en un número elevado de situaciones. Algunas referencias que evalúan el comportamiento de estimadores mediante simulación Monte Carlo son Rao et al. (1990), Silva y Skinner (1995) y Muñoz y Rueda (2009). Respecto a estudios 
de simulación llevados a cabo en el contexto de los gráficos de control, destacamos las aportaciones de Li y Pu (2012) y Mahmoud et al. (2014).

\subsection{Medidas empíricas para evaluar los gráficos de control.}

Existen distintas medidas utilizadas para valorar el comportamiento de un gráfico de control (Chan et al., 1988; Chen, 1997; Mahmoud et al., 2014; Montgomery, 2009). En este trabajo se emplea la medida más utilizada y conocida para analizar el rendimiento de los gráficos de control: la longitud media de las rachas en un gráfico de control, que denotaremos como $A R L$ (acrónimo de su denominación en la lengua inglesa: Average Run Length). La $A R L$ indica el número medio de puntos que se trazan en un gráfico de control antes de que un punto se encuentre fuera de los límites de control (Abbas et al., 2011; Chen, 1997; Jensen et al., 2006, Jones et al., 2001). Cuando el proceso está en control, la $A R L$ se puede definir como:

$$
A R L=\frac{1}{\alpha}=\frac{1}{1-P\left(L I C<\bar{x}_{i}<L S C\right)}
$$

donde

$$
\alpha=P\left(\text { rechazar } H_{0} \mid H_{0} \text { es cierta }\right)=1-P\left(L I C<\bar{x}_{i}<L S C\right)
$$

es la probabilidad de cometer un error de Tipo I asociado al siguiente test de hipótesis:

$$
\left\{\begin{array}{l}
H_{0}: \text { El proceso está bajo control } \\
H_{1}: \text { El proceso está fuera de control }
\end{array}\right.
$$

En el caso de límites de control seleccionados mediante el método USA (a una distancia de la línea media de 3 veces la desviación típica del estadístico) y cuando el proceso está bajo control, es conocido que $\alpha=0.0027$ y $A R L=370.4$. Que $\alpha=0.0027$ indica que el $0.27 \%$ de los puntos que estén fuera de las líneas de control serán falsas alarmas y no tienen una causa asignable de variación. Por su parte, $A R L=370.4$ indica que en media, habrá un punto fuera de control de cada 370 muestras, aproximadamente.

Cuando la media o la desviación típica de proceso (o ambos parámetros al mismo tiempo) cambian o se desplazan respecto a los valores deseables ( $\mu$ y $\sigma$, respectivamente) se dice que el proceso está fuera de control. En este caso se dice que el proceso se desplaza y/o cambia a un estado con media $\mu_{0} \mathrm{y}$ desviación estándar $\sigma_{0}$. En esta situación de fuera de control la $A R L$ se define como

$$
A R L=\frac{1}{1-\beta}=\frac{1}{1-P\left(L I C<\bar{x}_{i}<L S C \mid \mu_{0}, \sigma_{0}\right)}
$$

donde

$$
\beta=P\left(\text { No rechazar } H_{0} \mid H_{0} \text { es falsa }\right)=P\left(L I C<\bar{x}_{i}<L S C \mid \mu_{0}, \sigma_{0}\right)
$$
definido.

es la probabilidad de cometer un error de Tipo II en el contraste de hipótesis anteriormente

Basándonos en la definición de $A R L$, a continuación se plantean dos medidas empíricas para analizar el rendimiento o comportamiento de gráficos de control. Estas medidas se basan en el estudio de simulación Monte Carlo que se describe a continuación. En primer lugar, supongamos que el proceso está bajo control, es decir, la característica de calidad $x$ tiene media $\mu$ y desviación típica $\sigma$. En cada 
iteración del estudio de simulación los límites de control se estiman (es la denominada fase I del gráfico de control) seleccionando $m$ muestras con tamaño $n$ a partir de la correspondiente distribución con media $\mu$ y desviación típica $\sigma$. Una vez estimados y fijados los límites de control, en la fase II se seleccionan $D_{I I}$ muestras con tamaño $n$ y con la misma distribución que la utilizada en la fase I, es decir, también se asume que el proceso está bajo control en la fase II. En esta segunda fase se estudia el número de muestras que quedan fuera de los límites de control estimados en la fase I. Este proceso se repite $D_{I}$ veces.

Asumiendo que los parámetros $\mu$ y $\sigma$ son desconocidos, la primera medida empírica $\left(A R L_{1}\right)$ está directamente relacionada con la definición de $A R L$ dada por las ecuaciones (5) y (6). Sea $B_{i j}$ la variable que denota si la $j$-ésima muestra, con $j=1, \ldots, D_{I I}$, en la $i$-ésima iteración, con $i=1, \ldots, D_{I}$, se encuentra o no entre los límites de control, es decir, $B_{i j}=1$ si $L \hat{I} C_{i}<\bar{x}_{j}<L \hat{S} C_{i}$, y en caso contrario $B_{i j}=0$. Siguiendo la definición de $A R L$ dada por las ecuaciones (5) y (6) se define la medida empírica:

$$
A R L_{1}=\frac{1}{1-E[B]}
$$

donde $E[B]$ es el número medio de muestras que están bajo control, y cuya definición viene dada por:

$$
E[B]=\frac{1}{D_{I}} \sum_{i=1}^{D_{I}} E\left[B_{i}\right]=\frac{1}{D_{I}} \sum_{i=1}^{D_{I}} \frac{1}{D_{I I}} \sum_{j=1}^{D_{I}} B_{i j}=\frac{1}{D_{I} D_{I I}} \sum_{i=1}^{D_{I}} \sum_{j=1}^{D_{I I}} B_{i j}
$$

La segunda medida se define como la longitud media empírica de las rachas observadas en el estudio de simulación Monte Carlo. Matemáticamente esta expresión viene dada por:

$$
A R L_{2}=\frac{1}{D_{I}} \sum_{i=1}^{D_{I}} \frac{1}{r_{i}} \sum_{j=1}^{r_{i}} L_{i j}=\frac{1}{D_{I}} \sum_{i=1}^{D_{I}} \frac{1}{r_{i}} D_{I I}=\frac{D_{I I}}{D_{I}} \sum_{i=1}^{D_{I}} \frac{1}{r_{i}}
$$

donde $r_{i}$ es el número de rachas en la $i$-ésima iteración y $L_{i j}$ es la longitud de la $j$-ésima racha en la $i$-ésima iteración. Una racha se define como el número de puntos que trazan en el gráfico de control antes de que un punto caiga fuera de los límites de control.

Asumiendo que el proceso está bajo control, las medidas empíricas $A R L_{1}$ y $A R L_{2}$ anteriormente definidas deberían estar próximas a 370.4, el valor teórico de la medida $A R L$ definida en la ecuación (5).

En el caso de que el proceso se encuentre fuera de control, las muestras de la fase II se generan a partir de la correspondiente distribución, pero con media $\mu_{0} \mathrm{y}$ desviación típica $\sigma_{0}$. En esta situación las medidas $A R L_{1}$ y $A R L_{2}$ se definen de manera similar.

\subsection{Descripción de las situaciones que se analizan.}

En la sección anterior se ha detallado el estudio de simulación Monte Carlo que se ha llevado a cabo en este trabajo. Son varias las situaciones que se pretenden analizar. El objetivo de esta sección es describir los distintos escenarios que se van a estudiar mediante simulación en este trabajo.

En primer lugar, recordamos que es común considerar, tanto en la literatura como en la práctica, que la característica de calidad $x$ tiene distribución Normal. En este trabajo se asume, como es razonable, que $x$ sigue una distribución Normal. Por tanto, generaremos valores de una distribución 
Normal con media 10, lo que simulará que la media del proceso es $\mu=10$, mientras que los valores considerados para la desviación típica del proceso serán $\sigma=\{1,3\}$.

Por otro lado, se utilizará la distribución Gamma para el análisis del incumplimiento de la hipótesis de normalidad en los gráficos del control de la media. Con el fin de crear situaciones comparables en una mayor medida con la distribución Normal, se generan datos a partir de distribuciones Gamma con la misma media y mismos valores de la desviación típica que en el caso de la distribución Normal. En particular, se utilizan los parámetros de la distribución Gamma $\alpha=100 \mathrm{y}$ $\theta=0.1$ para generar valores con media 10 y desviación típica 1 , y parámetros $\alpha=100 / 9$ y $\theta=0.9$ para generar valores con media 10 y desviación típica 3 . Cabe recordar que la media y varianza de la distribución Gamma son $\alpha \theta$ y $\alpha \theta^{2}$ respectivamente.

Como un caso extremo ante la falta de normalidad utilizaremos la distribución Uniforme. Para este trabajo se han generado valores mediante una distribución Uniforme dentro del intervalo (8.268, 11.732), que se han seleccionado con el fin de obtener, de nuevo, una distribución con media $10 \mathrm{y}$ desviación típica 1. Cabe recordar que la media y varianza de la distribución Uniforme son $a+b / 2 \mathrm{y}$ $(b-a)^{2} / 12$, respectivamente.

Tal y como se ha descrito en la sección anterior, el estudio de simulación está basado en $D_{I}$ réplicas donde se obtienen límites de control y en cada una de estas réplicas se extraen $D_{I I}$ muestras. En este estudio de simulación se considera $D_{I}=D_{I I}=1000$. Los límites de control se obtienen a partir de $m$ muestras, considerando que $m=\{10,20,30,50,100,200,500,1000\}$. Por su parte, las muestras se seleccionan con tamaño $n$, donde $n$ se toma con valores desde 3 hasta 25. En la práctica es común considerar valores de $m$ entre 20 y 30 y valores de $n$ de al menos 5 unidades.

Para finalizar, cabe destacar que el estudio de simulación se ha llevado a cabo tanto para el caso en el que el proceso está bajo control como cuando tal proceso se encuentra fuera de control. Cuando el proceso está bajo control ya se ha comentado que las distintas muestras se generan a partir de distribuciones con media $\mu$ y desviación típica $\sigma$. En este trabajo también se analiza el incumplimiento de la hipótesis de normalidad cuando el proceso se encuentra fuera de control en la fase II. Los casos en los que se asume que el proceso se encuentra fuera de control son: (i) la media del proceso ha sufrido un cambio, (ii) la desviación típica del proceso ha sufrido un cambio y (iii) ambos parámetros del proceso han sufrido conjuntamente un cambio. En particular, es común considerar que este cambio viene dado para la media por $\mu_{0}=\mu+a \sigma$, mientras que el cambio en la desviación típica viene dado por $\sigma_{0}=b \sigma$, donde $a$ y $b$ son constantes. Podemos observar que el proceso estará bajo control cuando $a=0$ y $b=1$. El proceso sufrirá un cambio en la media cuando $a \neq 0$ y $b=1$, mientras que el proceso tendrá un cambio en la varianza o desviación típica cuando $a=0$ y $b \neq 1$. El proceso sufrirá un cambio en ambos parámetros en el resto de casos. En la Tabla 1 se resumen todos los casos analizados en este estudio de simulación respecto al estado del proceso.

Tabla 1. Diferentes valores para las constantes a y b y situación en la que se encuentra el proceso en cada caso.

\begin{tabular}{|c|c|c|c|}
\hline & \multicolumn{3}{|c|}{$\mathrm{b}$} \\
\hline $\mathrm{a}$ & 1.0 & 1.2 & 1.4 \\
\hline 0.0 & Bajo control & Cambio en la varianza & Cambio en la varianza \\
\hline 0.3 & Cambio en la media & Cambio en ambos parámetros & Cambio en ambos parámetros \\
\hline 0.6 & Cambio en la media & Cambio en ambos parámetros & Cambio en ambos parámetros \\
\hline \multicolumn{4}{|c}{ Fuente: Elaboración propia. }
\end{tabular}


En todos los escenarios descritos asumiremos que los parámetros del proceso son desconocidos y, por esta razón, se recurre a la fase I para la estimación de tales parámetros. Como estimador de la media del proceso $\mu$ utilizaremos el estimador $\overline{\bar{x}}$ definido en la ecuación (3). Por su parte, como estimador de la desviación típica del proceso $\sigma$ es común considerar los rangos muestrales, por lo que utilizaremos $\hat{\sigma}_{R}$, definido en la ecuación (4) como estimador del parámetro $\sigma$. En cualquier caso, cabe destacar que las conclusiones no difieren en gran medida si en lugar de considerar este estimador se utiliza cualquiera de los estimadores definidos en la ecuación (4).

\section{Resultados.}

En esta sección se muestran los resultados más relevantes derivados del estudio de simulación. Se distinguen los resultados obtenidos cuando el proceso está bajo control y cuando el proceso está fuera de control en la fase II. Recordamos que en ambos casos suponemos que el proceso está bajo control en la fase I, en la que se estiman los límites de control.

\subsection{Resultados empíricos cuando el proceso está bajo control.}

En las Figuras 2, 3, 4 y 5 se analiza la evolución del comportamiento de los distintos gráficos de control en términos de las medidas empíricas $A R L_{1}$ y $A R L_{2}$ y en las distintas situaciones descritas con anterioridad. Cabe recordar que los valores de $A R L_{1}$ y $A R L_{2}$ deberían estar próximos a 370.4, que es el valor teórico de la medida $A R L$ definida por la ecuación (5). En términos generales, observamos que la medida $A R L_{1}$ se acerca más a 370.4 que la medida $A R L_{2}$ a medida que aumentamos el tamaño de las muestras, lo que es razonable. Este hecho hace pensar que la medida $A R L_{1}$ puede ser mejor opción que $A R L_{2}$ para evaluar el comportamiento de los gráficos de control.

Como era de esperar, la distribución Uniforme destaca por obtener resultados alejados del valor teórico $A R L=370.4$. Por tanto, es de esperar que para el caso de distribuciones con una evidente falta de normalidad, como es el caso de la distribución Uniforme, el gráfico de control basado en la hipótesis de normalidad no sea una herramienta óptima para controlar la característica de calidad.

Con el fin de analizar mejor las diferencias existentes entre datos generados de una distribución Normal y datos generados de una distribución Gamma, se presentan las Figuras 2 y 3, en las que se eliminan los resultados obtenidos de la distribución Uniforme. En las Figuras 2 y 3 se considera el caso $\sigma=1$. Para valores pequeños de $m$ (Figura 2 con $m \leq 50$ ) se puede observar que los gráficos de control aplicados sobre distintas poblaciones tienen un comportamiento muy similar. Sin embargo, a medida que aumentamos el valor de $m$ (Figura 3 ) los valores obtenidos a partir de la distribución Gamma son, en general, ligeramente peores en comparación con los datos generados a partir de la distribución Normal, es decir, asumiendo normalidad se obtienen valores más próximos a 370.4 , aunque la diferencia no es relevante.

Es posible que la escasa diferencia entre los resultados derivados de las distribuciones Normal y Gamma se deba a que la distribución Gamma considerada no tenga demasiada asimetría $(\sigma=1)$. Sin embargo, cuando aumentamos el grado de asimetría (Figura 4 con $\sigma=3$ ) se aprecia que los datos procedentes de una distribución Normal tienen un comportamiento considerablemente mejor en comparación con los datos procedentes de la distribución Gamma. Esta propiedad se pone de manifiesto para las dos medidas empíricas y para todos los tamaños muestrales considerados. 
Figura 2. Valores de $A R L_{1}$ y $A R L_{2}$ con datos generados de las distribuciones Normal y Gamma.
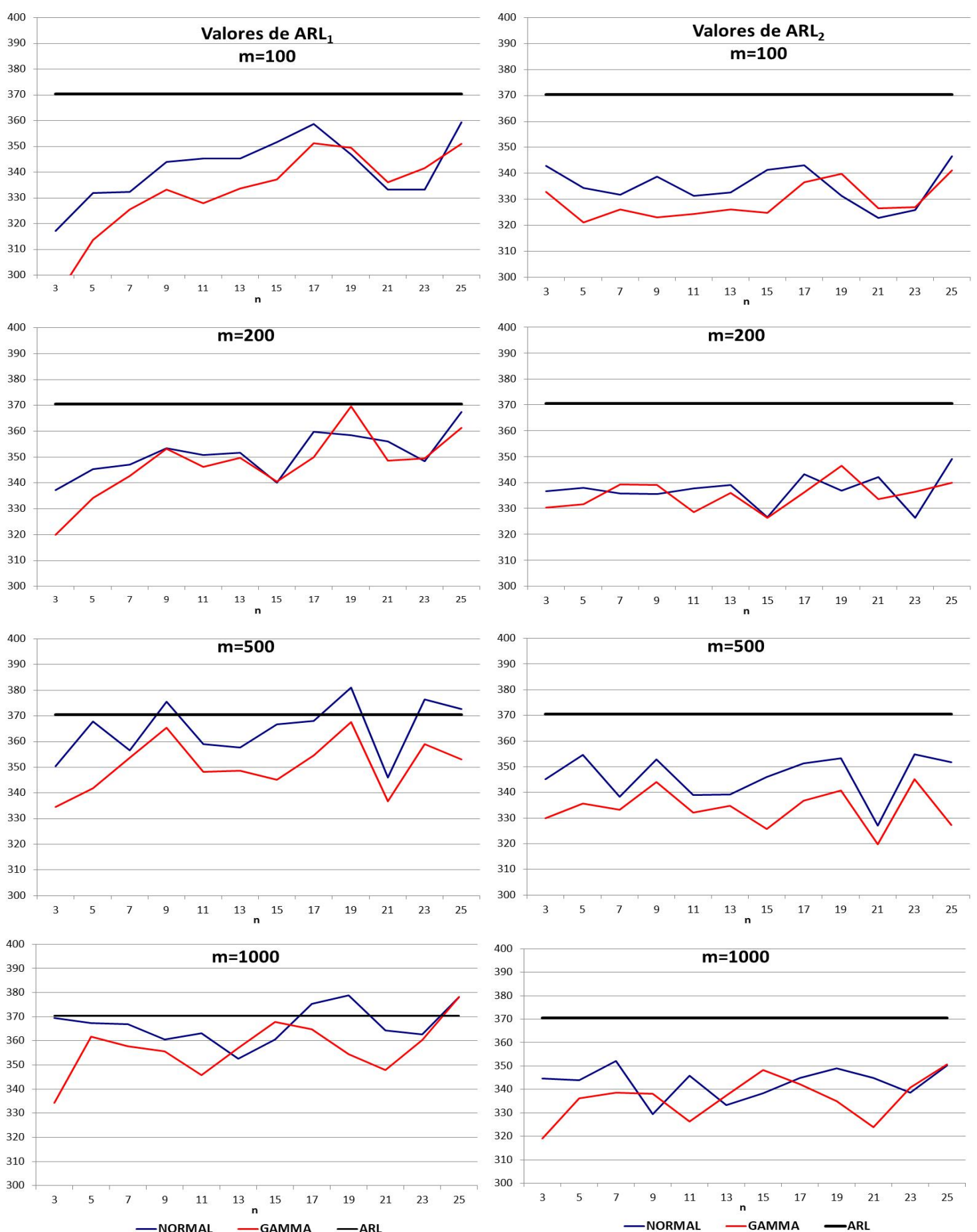

Se considera $\sigma=1$ y valores de $\mathrm{m}$ entre 10 y 50 . La línea continua negra representa el valor teórico de $A R L=370.4$.

Fuente: Elaboración propia. 
Figura 3. Valores de $A R L_{1}$ y $A R L_{2}$ con datos generados de las distribuciones Normal y Gamma.
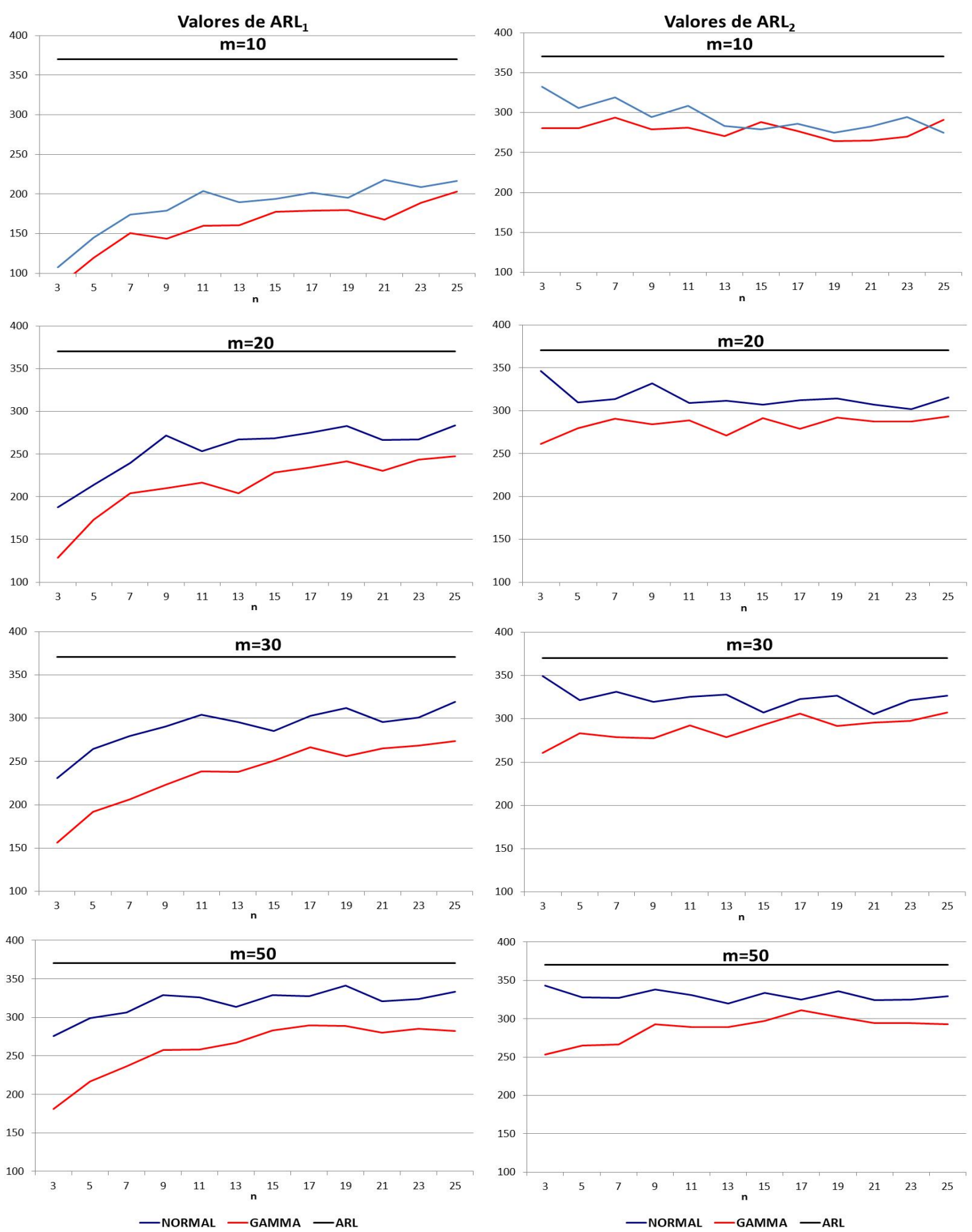

Se considera $\sigma=3$ y valores de $\mathrm{m}$ entre 10 y 50 . La línea continua negra representa el valor teórico de $\mathrm{ARL}=370.4$.

Fuente: Elaboración propia.

Aunque era razonable que la distribución Normal tuviese un mejor comportamiento que la distribución Gamma, este estudio de simulación también puede servir de ayuda para ver bajo qué condiciones el comportamiento asumiendo la distribución Gamma puede ser similar al observado bajo normalidad. Por ejemplo, si en la Figura 4 nos centramos en el caso con $m=30$ y $n=5$ podemos observar que el valor empírico de la medida $A R L_{1}$ se sitúa en torno al valor 270. La pregunta que nos podemos hacer en esta situación es qué valores de $m$ y $n$ se necesitarían para alcanzar un nivel similar 
de comportamiento en los datos generados a partir de la distribución Gamma. De este modo, si mantenemos fijo el valor de $m(m=30)$ observamos que se necesitarían muestras con un tamaño mínimo de $n=25$ para que los datos generados a partir de la distribución Gamma tuviesen un comportamiento similar, en términos de $A R L_{1}$, al de los datos de la distribución Normal cuando $m=30$ y $n=5$. Si optamos por fijar $n(n=5)$ para ambas poblaciones se observa a partir de la Figura 5 que se necesitaría un valor de $m$ mayor de 500 para alcanzar un valor de $A R L_{1}$ en torno a 270 .

Figura 4. Valores de $A R L_{1}$ y $A R L_{2}$ con datos generados de las distribuciones Normal y Gamma.
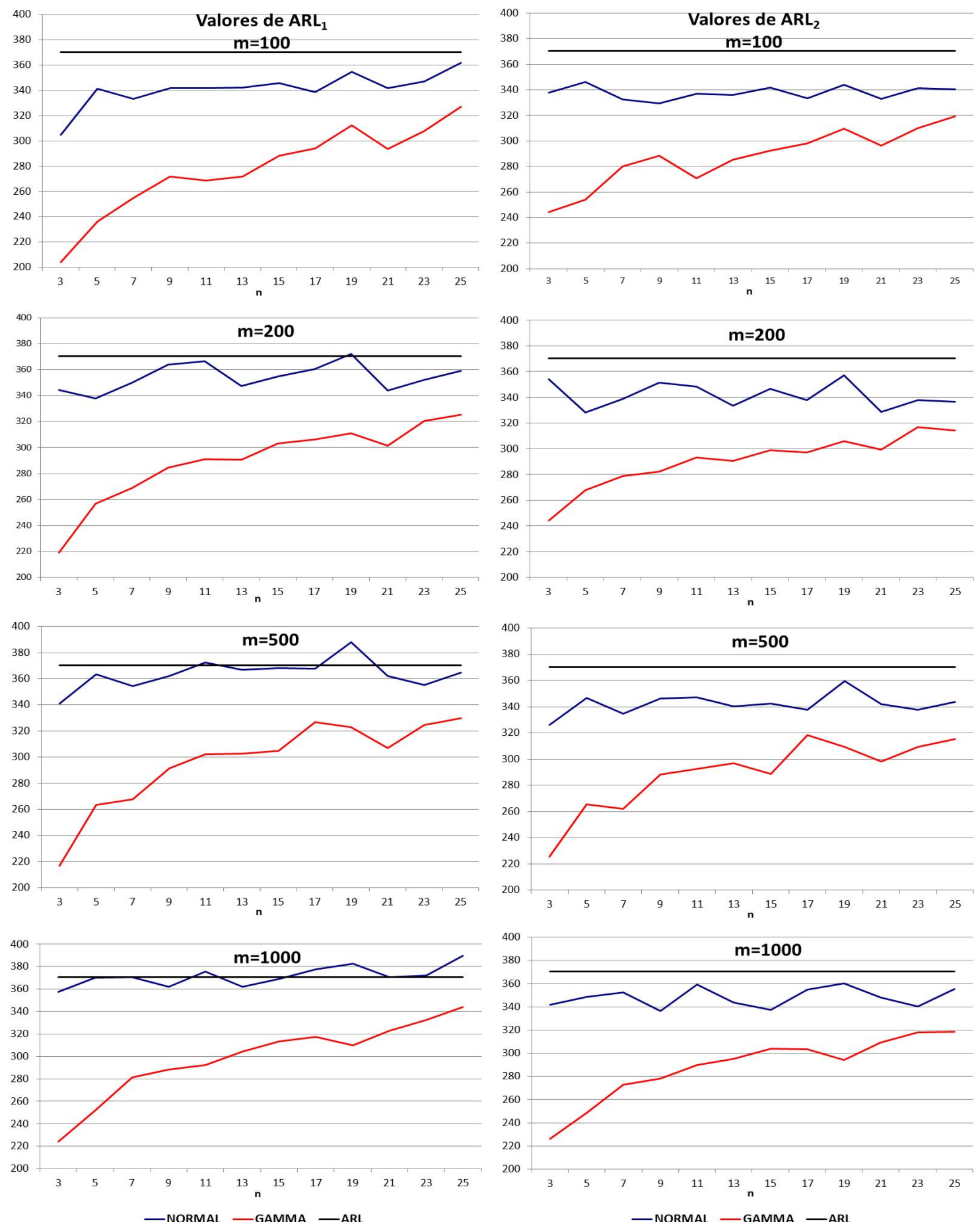
Se considera $\sigma=3$ y valores de $\mathrm{m}$ entre 100 y 1000 . La línea continua negra representa el valor teórico de $\mathrm{ARL}=$ 370.4 .

Fuente: Elaboración propia.

Figura 5. Valores de $A R L_{1}$ con datos generados de las distribuciones Normal y Gamma cuando $\sigma=\mathbf{1}$
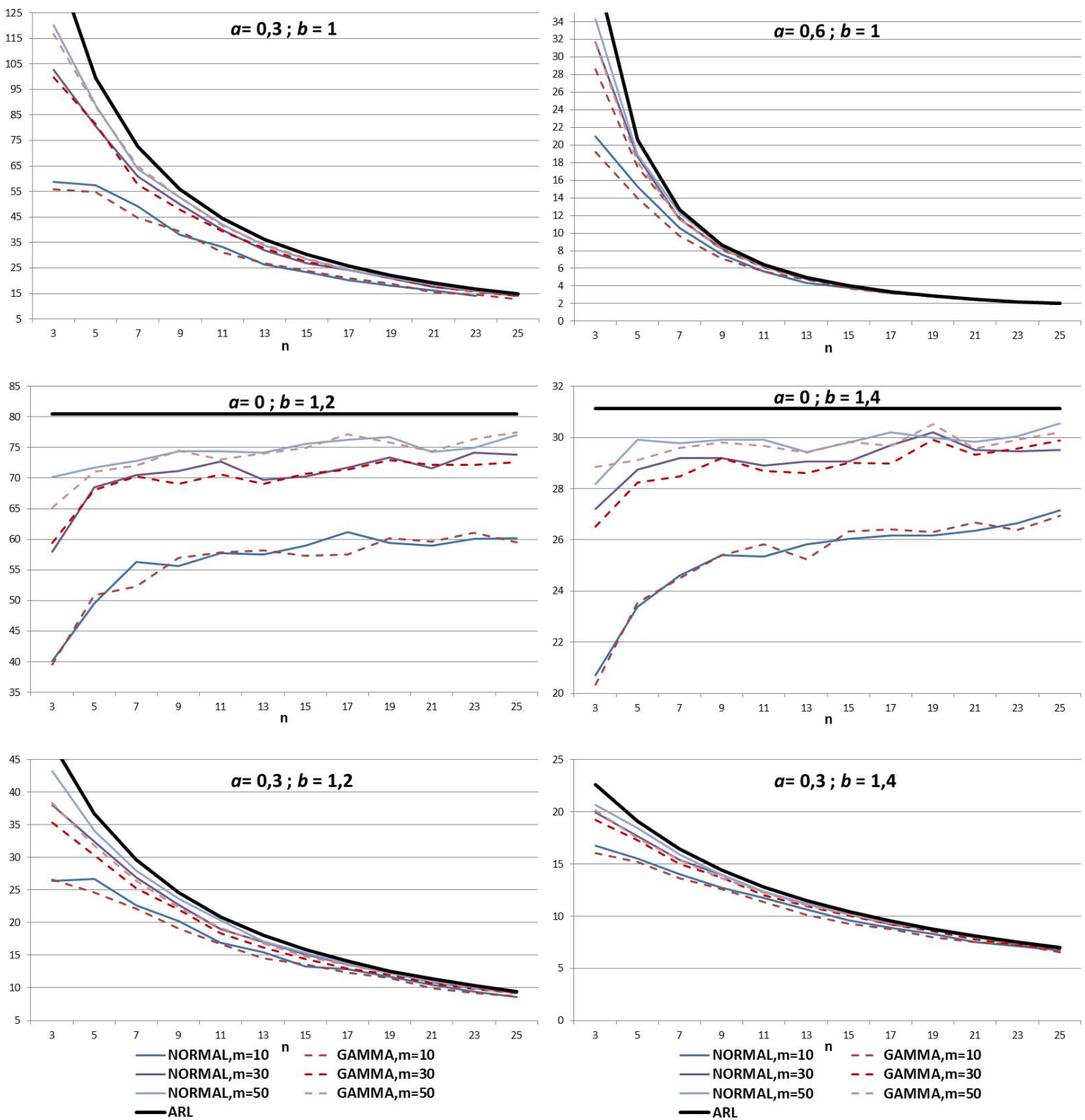

El proceso ha sufrido un cambio tanto en la media como en la desviación típica.

Fuente: Elaboración propia.

\subsection{Resultados empíricos cuando el proceso está fuera de control.}

Ahora analizamos el rendimiento de los gráficos de control cuando el proceso está fuera de control. Cabe recordar que la Tabla 1 resume los diferentes escenarios que analizamos en este estudio.

Cuando el proceso está fuera de control se puede ver fácilmente que el valor del error tipo II, $\beta$, se puede expresar como: 


$$
\beta=\phi\left(\frac{3-a \sqrt{n}}{b}\right)-\phi\left(\frac{-3-a \sqrt{n}}{b}\right)
$$

y, por tanto, el valor teórico de $A R L$ definido en la expresión (6) se puede obtener fácilmente. $\phi(\cdot)$ denota la función de distribución de una variable aleatoria $N(0,1)$.

En la Figura 6 se muestran los principales resultados. Por razones de claridad en los gráficos se ha utilizado únicamente la medida empírica $A R L_{1}$, puesto que es la que da una mejor aproximación. Además, solo se consideran los tamaños muestrales $m=\{10,30,50\}$ puesto que son los más comunes en la práctica. La distribución Uniforme no está incluida en la Figura 6 porque tiene un comportamiento muy extremo, distorsionando las figuras y dificultando su análisis.

Cuando el proceso tiene un cambio en la media $(a \neq 0$ y $b=1)$ y en la media y la desviación típica ( $a \neq 0$ y $b \neq 1$ ) se observa que la medida empírica $A R L_{1}$ está más cerca del valor teórico de $A R L$ a medida que los valores de $m$ y $n$ van aumentando. Por último, cuando el proceso tiene un cambio en la desviación típica ( $a=0$ y $b \neq 1$ ) hay una mejora en el comportamiento de los gráficos de control a medida que aumenta $m$. En todos los casos se observa que los gráficos de control basados en normalidad son ligeramente mejores que los gráficos de control basados en la distribución Gamma.

\section{Conclusiones.}

Los gráficos de control de la media (denominados internacionalmente como $\bar{X}$ charts) son una herramienta muy común para controlar la calidad de los procesos de fabricación. En la práctica la media real del proceso $(\mu)$ y la desviación típica real del proceso $(\sigma)$ son desconocidas. Por este motivo tenemos que estimarlas a partir de las expresiones dadas en las ecuaciones (3) y (4). Estas estimaciones se utilizan para obtener los límites de control del gráfico de control de la media, que están definidos en la ecuación (2). Cabe recordar que se obtienen límites de control a una distancia de la media de $\pm 3 \sigma$, lo que implica que el 99,73\% de los puntos deberían estar entre los límites de control. El 0,27\% restante son puntos que indicarían falsas alarmas, es decir, no tienen una causa asignable de variación porque es debida al azar. Por último, el valor teórico de $A R L$ en esta situación es 370.4, es decir, en media debería situarse un punto fuera de control cada 370 muestras, aproximadamente.

El supuesto de normalidad en los gráficos de control significa que la función de densidad de la característica de calidad $x$ tiene que ser normal. Sin embargo, esta hipótesis puede no darse debido a la naturaleza del proceso, como por ejemplo procesos químicos, que a menudo son sesgados.

El principal objetivo de este trabajo es analizar, mediante la técnica de simulación Monte Carlo, el efecto del incumplimiento de la hipótesis de normalidad en los gráficos de control de la media, ya que la falta de normalidad puede reducir la capacidad de que el gráfico de control de la media detecte cambios debidos a causas asignables.

En este trabajo se definen las medidas empíricas $A R L_{1}$ y $A R L_{2}$, que se basan en la definición teórica de $A R L$. Con la simulación Monte Carlo se estudia el rendimiento de los gráficos dependiendo de la distribución que presente nuestro proceso. En términos generales, la simulación Monte Carlo indica que el rendimiento de los gráficos de control de la media es mejor con los valores obtenidos con la medida empírica $A R L_{1}$ y a medida que aumenta el número de muestras $(m)$ y el tamaño de muestra (n). Hay que destacar que para situaciones en las que la característica de la calidad tenga una distribución Uniforme no se aconseja usar estos gráficos basados en normalidad, ya que se obtendrían resultados muy poco fiables. Sin embargo, para distribuciones con leves asimetrías obtenemos evidencias de que los gráficos de control de la media son una herramienta que se podría utilizar para controlar la media del proceso. Habría que aumentar levemente los tamaños muestrales de $n$ o de $m$ para 
alcanzar un nivel de $A R L$ similar al obtenido bajo normalidad. Para distribuciones un poco más asimétricas el comportamiento de los gráficos de control es aceptable, aunque evidentemente son necesarias muestras con tamaños más elevados para alcanzar buenos niveles de $A R L$. Las figuras presentadas en este trabajo pueden ayudar a realizar este tipo de comparaciones. Por último, cabe destacar que los distintos estudios de simulación se han realizado tanto en el caso de que el proceso se encuentre bajo control en la fase II como en una situación de fuera de control.

\section{Financiación}

Esta investigación ha sido parcialmente apoyada por el Ministerio de Economía, Industria y Competitividad, la Agencia Estatal de Investigación (ASI) y el Fondo Europeo de Desarrollo Regional (FEDER) (referencia del proyecto ECO2017-86822-R).

\section{Referencias}

Abbas, N., Riaz, M., \& Does, R.J. (2011). Enhancing the performance of EWMA charts. Quality and Reliability Engineering International, 27(6), 821-833.

Chan, L.K., Hapuarachchi, K.P., \& Macpherson, B.D. (1988). Robustness of $\bar{X}$ and $R$ charts. IEEE Transactions on reliability, 37(1), 117-123.

Chen, G. (1997). The mean and standard deviation of the run length distribution of X charts when control limits are estimated. Statistica Sinica, 7(3), 789-798.

Jensen, W.A., Jones-Farmer, L.A., Champ, C.W., \& Woodall, W.H. (2006). Effects of parameter estimation on control chart properties: A literature review. Journal of Quality Technology, 38(4), 349-364.

Jones, L.A., Champ, C.W., \& Rigdon, S.E. (2001). The performance of exponentially weighted moving average charts with estimated parameters. Technometrics, 43(2), 156-167.

Li, Y., \& Pu, X. (2012). On the performance of two-sided control charts for short production runs. Quality and Reliability Engineering International, 28(2), 215-232.

Mahmoud, M.A., Saleh, N.A., \& Madbuly, D.F. (2014). Phase I analysis of individual observations with missing data. Quality and Reliability Engineering International, 30(4), 559-569.

Mitra, A. (2016). Fundamentals of quality control and improvement. Hoboken, N.J.: Wiley.

Montgomery, D.C. (2009). Statistical quality control (6 ${ }^{\text {th }}$ ed.). New York: Wiley.

Muñoz, J.F., \& Rueda, M. (2009). New imputation methods for missing data using quantiles. Journal of Computational and Applied Mathematics, 232(2), 305-317.

Rao, J.N.K., Kovar, J.G., \& Mantel, H.J. (1990). On estimating distribution functions and quantiles from survey data using auxiliary information. Biometrika, 77(2), 365-375.

Saleh, N.A., Mahmoud, M.A., Keefe, M.J., \& Woodall, W.H. (2015). The difficulty in designing Shewhart X and X control charts with estimated parameters. Journal of Quality Technology, 47(2), $127-138$. 
Schilling, E.G., \& Nelson, P.R. (1976). The effect of non-normality on the control limits of X charts. Journal of Quality Technology, 8(4), 183-188.

Scrucca, L. (2004). qcc: An R package for quality control charting and statistical process control. $R$ News, 4(1), 11-17.

Shewhart, W.A. (1931). Economic control of quality of manufactured product. New York: Van Nostrand.

Silva, P.N., \& Skinner, C.J. (1995). Estimating distribution functions with auxiliary information using poststratification. Journal of Official Statistics, 11(3), 277-294.

Woodall, W.H., \& Montgomery, D.C. (2000). Using ranges to estimate variability. Quality Engineering, 13(2), 211-217.

Yourstone, S. A., \& Zimmer, W. J. (1992). Non-normality and the design of control charts for averages. Decision Sciences, 23(5), 1099-1113. 